\title{
Oxygen Spectroscopy Laser Sounding Instrument for Remote Sensing of Atmospheric Pressure ${ }^{12}$
}

\author{
Mark A. Stephen \\ NASA - Goddard Space Flight Center \\ Code 554 \\ Greenbelt, MD 20771 \\ 301-614-6737 \\ mark.a.stephen@nasa.gov
}

\author{
S. Randy Kawa \\ NASA - Goddard Space Flight Center \\ Code 613.3 \\ Greenbelt, MD 20771 \\ 301-614-6004 \\ Stephan.R.Kawa@gsfc.nasa.gov
}

\author{
Jianping Mao . \\ RSIS \\ Code 610.2 \\ Greenbelt, MD 20771 \\ 301-614-6079 \\ jmao@pop600.gsfc.nasa.gov
}

Xiaoli Sun

NASA - Goddard Space Flight Center Code 694

Greenbelt, MD 20771

301-614-6737

Xiaoli.Sun@gsfc.nasa.gov

\author{
James B. Abshire \\ NASA - Goddard Space Flight Center \\ Code 690 \\ Greenbelt, MD 20771 \\ 301-614-6081 \\ James.B.Abshire.1@gsfc.nasa.gov
}

\author{
Michael A. Krainak \\ NASA - Goddard Space Flight Center \\ Code 554 \\ Greenbelt, MD 20771 \\ 301-286-4881 \\ mkrainak@pop500.gsfc.nasa.gov
}

Abstract-We report on the progress of an Oxygen spectroscopy laser sounding instrument designed as a calibration channel for a carbon dioxide $\left(\mathrm{CO}_{2}\right)$ laser sounding instrument. We have developed a pulsed, frequency-doubled, fiber laser transmitter for use in an oxygen instrument. The instrument concept uses the pressure broadening of spectroscopic lines of the diatomic oxygen A-band to deduce atmospheric pressure.

There are many uses for this measurement but we are developing it primarily to make a measurement of the dry mixing ratio of $\mathrm{CO}_{2}$. The $\mathrm{CO}_{2}$ measurement can be affected by changes in atmospheric properties such as humiditiy, temperature and pressure. To remove these variances requires measuring a stable, well-mixed gas like Oxygen.

We will report on the basic theory behind the instrument, measurements made at a test site at Goddard, review the current state of the instrument technologies and the necessary steps to bring them to space readiness, and review the current state of the instrument development.

\section{TABLE OF CONTENTS}

1. INTRODUCTION 1

2. THEORETICAL ANALYSIS ..................................... 2

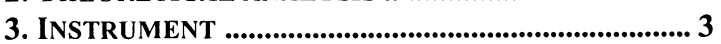

4. CONClUSIONS …........................................................... 5

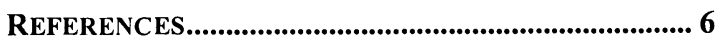

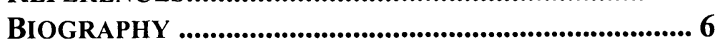

$1-1$ U.S. Government work not protected by U.S. copyright
${ }^{2}$ IEEEAC paper \#1465, Version 3, Updated December 13, 2007

\section{INTRODUCTION}

This research is directed toward developing a remote sensing instrument capable of measuring atmospheric pressure. The primary focus for the authors is as a calibration channel for a carbon dioxide $\left(\mathrm{CO}_{2}\right)$ sensor, however there are many other uses for such an instrument, including: weather prediction, atmospheric modeling and dynamic gravity field measurements.

The $\mathrm{CO}_{2}$ measurement of scientific interest is the fraction of $\mathrm{CO}_{2}$ compared to the dry atmosphere - the so-called dry mixing ratio (DMR) - usually expressed in percentage or parts per million (ppm) in the case of $\mathrm{CO}_{2}$. Because diatomic oxygen $\left(\mathrm{O}_{2}\right)$ has a constant dry mixing ratio of $20.95 \%$, it is an ideal reference for measuring the variability of a gas like $\mathrm{CO}_{2}$.

If one just measured the total number of $\mathrm{CO}_{2}$ molecules in a certain volume of atmosphere, the biggest error sources would be the atmospheric pressure and water vapor variablility. The atmosphere is denser at higher pressures so more $\mathrm{CO}_{2}$ will be measured at higher pressures. Similarly, increased atmospheric water vapor concentration will change the absolute (but not relative) percentages of other molecules. Measuring the $\mathrm{CO}_{2}$ dry-mixing ratio by dividing by the $\mathrm{O}_{2}$ concentration excludes both of these effects. Unfortunately, direct measurement of the $\mathrm{O}_{2}$ concentration is difficult. Therefore we measure the atmospheric pressure and the water vapor concentration separately and deduce the 
$\mathrm{O}_{2}$ concentration. Adequate water vapor data is available from the Atmospheric Infra-red Sounder [1] so we focus on the atmospheric pressure measurement required.

We use spectroscopy of pressure broadened lines of the diatomic oxygen A-band as the basis for the measurement. Others have used similar methods of both passive [1] and active [2-5] spectroscopy with success. The oxygen dry mixing ratio stays constant at $20.95 \%$ so it makes a good reference for a variable gas like $\mathrm{CO}_{2}$. We are developing a measurement technique that capitalizes on the spectroscopy work of others but uses new laser and instrument architectures.

The laser developed is a frequency-doubled, DFB seeded erbium-doped fiber amplifier (EDFA). It is a tunable, narrow frequency, rugged, efficient, high peak power device capable of very high resolution spectroscopy. The details will be considered in a later section.

The instrument uses laser pulses reflecting from a hard target and measures the average atmospheric pressure of the column of air through which the laser pulses travel. This is in contrast to differential absorption LIDAR (DIAL) instruments that rely on atmospheric backscatter for their return signals. An instrument using a hard target return requires several orders of magnitude less transmitted laser power than one relying on atmospheric backscatter. This technique does not resolve range like a LIDAR instrument, however for atmospheric pressure, whose vertical distribution is well approximated by the hydrostatic relationship [6], this is not a substantial loss.

The spectroscopy method we employ is very similar to previous instruments. We will measure the absorption in the trough between two strongly absorbing lines in the oxygen A-band near $760 \mathrm{~nm}$. This instrument design and component technology is being considered to easily extend to both aircraft and satellite but our development to date has been ground-based.

First we will analyze the theory behind the measurement. We will discuss the spectrsoscopy and pressure broadening effects. In section 3 we will discuss the instrument design and performance and compare to theory.

\section{Theoretical ANALysis}

In order to measure the $\mathrm{CO}_{2}$ dry mixing ratio to a part per million (ppm), knowledge of the atmospheric pressure to $<3$ mbar is required. Korb et al measured atmospheric pressure to $1.5-2.0$ mbar accuracy [5] using DIAL in the oxygen A-band. We propose a similar instrument but employing a different laser technology.

Using oxygen for this measurement is beneficial for several reasons. First, oxygen is well-mixed, both temporally and spatially, in the atmosphere so the concentration is constant and known. The oxygen A-band absorption near $760 \mathrm{~nm}$ is free from other atmospheric absorptions that could confuse or corrupt the measurements. The previous work in this spectral region proves the feasibility and the strength of the absorptions are also convenient to work with over the path lengths required as discussed in more detail later.

The transmission through the atmosphere is given by Beer's law:

$$
T(w)=\exp [-K(w) \cdot L]
$$

Where $\mathrm{L}$ is the path length of the light and $\mathrm{K}$, the resonant absorption is given by,

$$
K(w)=n \cdot S_{j} \cdot f
$$

where $S_{j}$ is the line strength parameter, $n$ is the number density of $\mathrm{O}_{2}$, defined in Eq. (3), and $\mathrm{f}$ is a lineshape function defined in Eqs. (4-6).

$$
n=q \cdot\left(1-q^{*}\right) \cdot \frac{P}{R \cdot T}
$$

In $\mathrm{Eq}$ (3), $\mathrm{q}$ is the dry mixing volume ratio of $\mathrm{O}_{2}$, equal to $20.95 \%, \mathrm{q}^{*}$ is water vapor concentration by volume, $\mathrm{P}$ is atmospheric pressure, $\mathrm{R}$ is the universal gas constant and $\mathrm{T}$ is the temperature of the atmosphere.

In order to calculate the expected absorption, we use a voigt line shape. This function is the convolution of Gaussian and Lorentzian line shapes and is used when both doppler and pressure broadening are significant. Doppler (or temperature) broadening is Gaussian and Collision (or pressure) broadening is Lorentzian. The voigt function is then defined by:

$$
f_{V}(w)=\int_{-}^{\bullet} f_{G}\left(w-w_{o}\right) \cdot f_{L}\left(w-w_{o}-\tau\right) \cdot d \tau
$$

where $w_{0}$ is the wavenumber of the absorption peak, $w$ is the wavenumber and $f_{G}$ and $f_{L}$ are the gaussian and lorentzian functions, respectively, defined as follows:

$$
\begin{gathered}
f_{G}(w)=\sqrt{\frac{\ln (2)}{\pi}} \cdot \frac{1}{\gamma_{G}} \cdot \exp \left[-\ln (2) \cdot \frac{\left(w-w_{o}\right)^{2}}{\gamma_{G}{ }^{2}}\right] \\
f_{L}(w)=\frac{1}{\pi} \cdot\left[\frac{\left(\gamma_{L} / 2\right)}{\left(w-w_{o}\right)^{2}+\left(\gamma_{L} / 2\right)^{2}}\right]
\end{gathered}
$$

Where $\gamma_{G}$ is the half width at half maximum (HWHM) gaussian linewidth and $\gamma_{\mathrm{L}}$ is the HWHM Lorentzian linewidth. The gaussian linewidth is defined, from Boltzman statististics [7], to be 


$$
\gamma_{G}=\frac{w_{o}}{c} \sqrt{2 \cdot R \cdot \ln (2) \cdot\left[\frac{T}{M}\right]}
$$

Where $\mathrm{c}$ is the speed of light and $\mathrm{M}$ is the molar mass of the oxygen molecule. While the Lorentzian linewidth is defined as

$$
\gamma_{L}\left(\omega_{o}\right)=\gamma_{\text {air }} \cdot P \cdot\left(\frac{T_{o}}{T}\right)^{n_{\text {ar }}}
$$

Where $\gamma_{\text {air }}$ is air-broadened HWHM at $\mathrm{T}_{\mathrm{o}}=296 \mathrm{~K}$ and $\mathrm{n}_{\text {air }}$ is the coefficient of temperature dependence of the airbroadened half-width. The values used in these calculations for $\mathrm{S}_{\mathrm{j}}, \gamma_{\text {air }}, \mathrm{n}_{\text {air }} \mathrm{w}_{\mathrm{o}}$ are from the HiTRAN [8], a database of measured and calculated parameters for atmospheric absorption spectra.

In the case of multiple lines with overlapping absorptions, Eq. (1) becomes

$$
T(w)=\exp \left\{-\left[K_{1}(w)+K_{2}(w)+\ldots K_{n}(w)\right] \cdot L\right\}
$$

Where $K_{1}, K_{2}, \ldots K_{n}$ are calculated individually from values listed in HiTRAN for each line of interest. (The theory for a calculation of two lines is illustrated in Figure 1 - inset.)

The technique we explore in this research uses the absorption between two strongly absorbing oxygen absorption lines. We use this measurement away from the peak absorption to isolate the pressure broadening in the absorption. Because there is appreciable doppler and pressure broadening in the oxygen lines of interest, we must distinquish between them. The doppler broadening has a Guassian shape whereas the pressure broadening has a Lorentzian shape. The lorentz function goes to zero much slower away from the absorption peak than does the Gaussian function. The line wing is therefore dominated by the pressure effect. Measuring the absorption between two closely spaced lines increases the amount of absorption from a single line, and decreases sensitivity to frequency drift [2].

The Figure 1 inset, is an example of an absorption calculation showing the transmission as a function of wavelength for the two lines of interest in the oxygen A-Band and the trough between them.

A modified version of the LIDAR equation, which calculates the column average due to a hard target return rather than range resolved atmospheric backscatter, is shown below.

$$
\begin{gathered}
E_{R x}(\lambda)=E_{T x}(\lambda) \cdot T_{B S} \cdot T_{c} \cdot T_{r}(\lambda) \cdot R_{\text {target }} \cdot \eta_{R x} \\
E_{M o n}(\lambda)=E_{T x}(\lambda) \cdot R_{B S} \cdot \eta_{M o n}
\end{gathered}
$$

In this equation, $E_{R x}$ is the energy received, $E_{M o n}$ is the energy measured at the monitor receiver, $\mathrm{E}_{\mathrm{Tx}}$ is the energy from the transmitter, $T_{B S}$ is the transmission of a beam splitter used to monitor the outgoing energy, $R_{B S}$ is the reflectivity of this beam splitter, $T_{c}$ is the transmission through the atmosphere that includes all continuum and scattering losses, $T_{r}$ is the resonant transmission, $\eta_{R x}$ is the efficiency of the main instrument receiver, while $\eta_{\text {Mon }}$ is the efficiency of the monitor receiver. (The term, $\eta_{R x}$, used to represent the efficiency of the receiver, includes many terms which are often written out explicitly like the area of the receiver telescope and the inverse square dependence on range. These terms have been consolidated into the receiver efficiency because they would unnecessarily complicate the discussion.)

It is easily shown using Eqs. $(10 \& 11)$ that, if a reference wavelength is chosen such that $\mathrm{T}_{\mathrm{r}}\left(\lambda_{\text {ref }}\right)$ equals one meaning no resonant absorption at that wavelength - then the resonant transmission can be measured as follows:

$$
T_{r}(\lambda)=\frac{E_{R x}(\lambda) / E_{\text {Mon }}(\lambda)}{E_{R x}\left(\lambda_{\text {ref }}\right) / E_{\text {Mon }}\left(\lambda_{\text {ref }}\right)}
$$

This assumes that the only terms affected by a change in wavelength over the small tuning range (of $\sim 200 \mathrm{pm}$ ) are those with the dependence written explicity. The degree to which this is true will largely determine the accuracy and effectiveness of the instrument.

The pressure dependence of the absorption trough is calculated from Eq. (9) using the two dominant lines and illustrated in Figure 1. The slope of $0.73 \%$ per mbar change in atmosphere is calculated from the result. In order to measure the $\mathrm{CO}_{2}$ dry-mixing ratio to $\sim 1 \mathrm{ppm}$, requires knowledge of the atmospheric pressure to an accuracy of better than 3 mbar. This leads to a measurement accuracy of the resonant transmission to $\sim 0.2 \%$. Although very challenging, analysis and measurements mentioned earlier demonstrate it is feasible. Further analysis of the error sources of this type of measurement is available [9]. An instrument capable of an atmospheric pressure measurement to this accuracy would find many applications in addition to $\mathrm{CO}_{2}$ calibration.

This trough between these two particular lines was chosen for several reasons. First, the line strengths of lines is temperature insensitive. This means that changes in temperature will have only small effects on the transmission spectrum. Also, the lines are strongly absorbing so the line wing still has a very appreciable absorption. Calculation of the absorption trough for a round trip path from a nadirviewing satellite has around $50 \%$ transmission which is optimum. In addition, this wavelength overlaps nicely our laser technology - a frequency doubled EDFA. This is in 
contrast to Korb et al [5] who used the $\mathrm{R}$ branch rather than the $\mathrm{P}$ branch of the oxygen A-band.

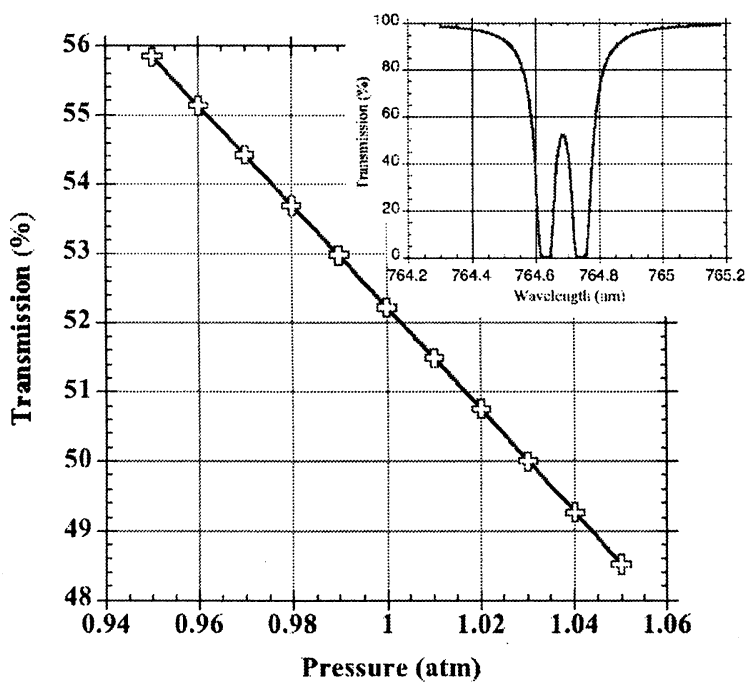

Figure 1 - Calculation of pressure dependence of the transmission through a $7 \mathrm{~km}$ horizontal path of atmosphere (round trip) at $764.7 \mathrm{~nm}$ (the trough between two strongly absorbing (and saturated) oxygen lines.) The inset shows the calculated transmission vs. wavelength at $1 \mathrm{~atm}$.

\section{INSTRUMENT}

For the space measurement, we have baselined a two spectral line instrument that measures only at a signal and a reference wavelength. However for the development of the instrument, tuning through the entire line is preferable in order to measure and assess noise sources and instrument uncertainties. The measurement technique is based on the fact that the only variation in wavelength response is from the absorption. However, if there are other instrument elements like etalon effects, errors will be introduced. Mathematically this can be seen if any of the terms in Eqs. $(10,11)$ have a wavelength dependence that is not explicitly written. If this is the case, then the division in Eq (12) will not correctly cancel these terms and unknown errors will persist. Scanning across the whole line will allow us to track these changes and reduce or eliminate them. This effect is a common one in high fidelity spectroscopy instruments $[10,11]$.

In the present instrument set-up, the pulses from the laser transmitter are directed at a cooperative target via a transmitter telescope which is co-aligned with a receiver telescope. The light travels across an open section of atmosphere, rebounds from the target and is then collected by the receiver. The measurement we have performed is across a 443 meter round trip horizontal open path. The instrument layout is illustrated in Figure 2.

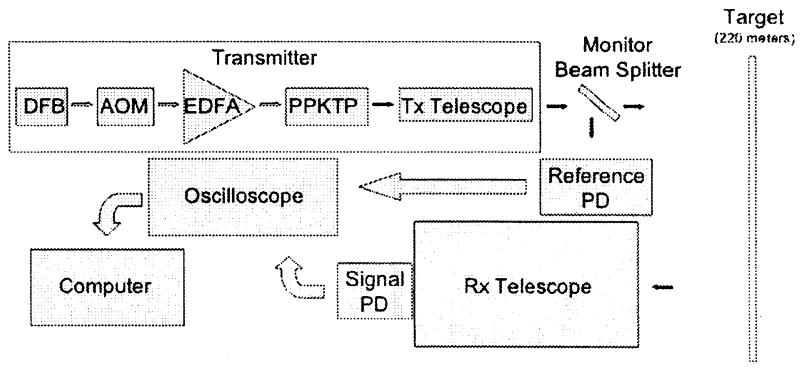

Figure 2 - Diagram of instrument set-up illustrating laser transmitter architecture, receiver telescope and laser propogation.

The laser transmitter is a DFB seeded, frequency doubled, EDFA. The basic architecure is shown in the transmitter box of Figure 2. The seed is a very narrow frequency $(\sim 1$ $\mathrm{MHz}$ ), wavelength tunable, DFB laser with a wavelength centered around $1530 \mathrm{~nm}$. The wavelength is tuned by changing the drive current applied to the seed laser. The output of the seed is then externally modulated with a fibercoupled acousto-optic modulator to yield transform limited, $200 \mathrm{~ns}$ laser pulses with $40 \mathrm{~dB}$ extinction ratio. These laser pulses are then coupled into an EDFA. The EDFA ouput is free-space coupled into a periodically-poled, KTP crystal to frequency double the fundamental to the $765 \mathrm{~nm}$ wavelength regime. We have reported on other versions of this transmitter operating at slightly different wavelengths in more detail. $[12,13]$

It is the development and application of this laser technology where we are making the largest contribution from previous measurements. The previous pressure measurements referenced above, used dye and alexandrite lasers, which are far less efficient, had more frequency instability, and were much more difficult to use and to ruggedize for airplane or satellite platforms. This transmitter is fiber-based and capitalizes on substantial device development and engineering from the telecommunications industry. The components are high performance and cost effective. Because most of the system is fiber coupled, alignment is inherently stable and the system is lightweight and efficient. The spectrum is very narrow and stable.

For Frequency doubling the output of the EDFA, we use a $30 \mathrm{~mm}$ long PPKTP crystal. Its full width at half Maximum wavelength bandwidth is $760 \mathrm{pm}$ so tuning at or above $90 \%$ of peak conversion is achieved over a tuning range of 260 pm. At high peak optical pump power, this will get even broader because of the saturation. After the doubling crystal, a long-wave pass filter separates the doubled frequency from the fundamental. The doubled light is then fiber coupled into the transmitter telescope. 
Part of the transmitted signal beam is picked off with a beam splitter and sent to a photo-detector to monitor the output. This signal is used to track the energy of each pulse sent out by the system. We use an integrating sphere to reduce signal variation due to spatial variations in the laser beam. The detector output is AC-coupled to an oscilloscope.

We use a ten inch Schmidt-Cassegrain telescope to collect the return signal. With custom mounts connected to the back end of the telescope, we used a fast lens to couple light into a fiber. This changes the telescope system from an $\mathrm{f} / 10$ to about an $\mathrm{f} / 1.5$ optical system. We are trying to optimize light collection so we used a large core $(600 \mu \mathrm{m})$ fiber with a numerical aperature of 0.37 . The fiber was then terminated in a large area PIN detector. The detector output was AC-coupled to an oscilloscope. The AC coupling allowed us to reduce the effect of the solar background which was larger than the signal.

The data was averaged on the oscilloscope and the magnitude (peak-to-valley) of the signal pulse was divided by the magnitude of the monitor pulse. Taking the ratio of the signal and the monitor eliminates the energy fluctuations from the transmission measurement as shown mathematically in Egs. (10-12). We then tuned the current of the DFB seed laser to tune the transmitter to a new wavelength and take another data point. In this way we measured the transmission of Figure 3, illustrated below.

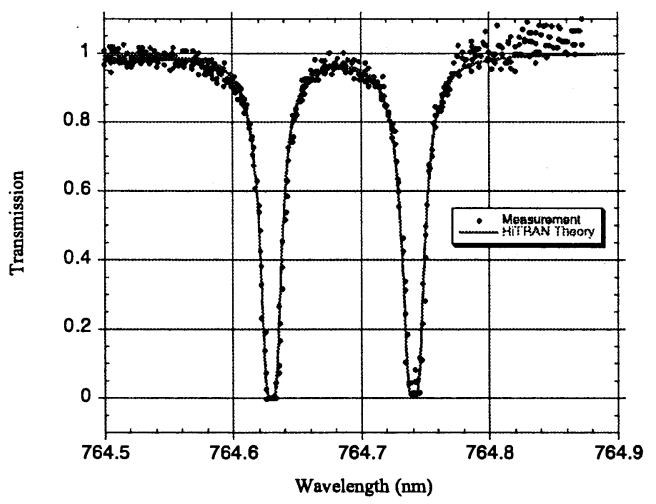

Figure 3 - Theory compared to measurement for the transmission through a 443 meter path. The scan was preformed by changing the drive current of the DFB diode seed laser.

Using equation (9), the parameters from HiTRAN and measured values of pressure, temperature and humidity from a coincident weather station, the theoretical transmission curve was calculated. (For a satellite version of the instrument, the temperature and water vapor concentration are available from other satellite measurements like Atmospheric Infraread Sounder [14].) The calculation is compared to the actual measurement of our bread board instrument with excellent agreement between them.

\section{Conclusions}

To date, all the basic principles necessary for this instrument have been demonstrated. Analysis and previous measurements demonstrate the instrument measurement accuracy is acheivable. Progress on the laser transmitter demonstrates all the fundamental performance elements can be realized. A preliminary instrument configuration has demonstrated the basic function of the system which has been used to measure the transmission through the atmosphere that shows the expected resonant absorption in the oxygen A-band.

There are several aspects of the measurement which still need to be optimized in order to realize the full potential of the instrument. The peak power of the transmitted signal was only about $50 \mathrm{~mW}$ due to peak power limitations in the present amplifier. We are currently improving the connector for this amplifier to allow higher peak powers but the power scaling above 100 watts has already been demonstrated in previous work [13]. For this measurement, the EDFA did not employ polarization maintaning fiber which helps minimize the frequency doubled energy. This is easily fixed and has been demonstrated in a different amplifier [12].

Because much of the effort has gone toward developing the laser transmitter, there are still many improvements to be made on the rest of the instrument. The oscilloscope used in this measurement can be replaced with a high speed Digital Acquistion Board and the data collection and processing can be greatly improved. This will lead to the ability to increase the speed of the scan which will help reduce noise sources do to atmospheric turbulence. The detectors are not optimized and will be replaced eventually with photon counters. An in-line solar filter will also greatly improve the signal-to-noise (SNR) ratio for the measurement. Longer path lengths will also increase the absorption in the trough and further increase the SNR at the wavelength of interest. Once the data collection has been improved, a more systematic analysis of the proper processing and averaging techniques can be explored.

We expect to continue to improve the instrument performance and make atmospheric pressure measurements and compare them to a coincident weather station to evaluate its accuracy. 


\section{REFERENCES}

[1] M. G. Divakarla, C. D. Barnet, M. D. Goldberg, L. M. McMillin, E. Maddy, W. Wolf, L. Zhou, X. Liu, "Validation of Atmospheric Infrared Sounder temperature and water vapor retrievals with matched radiosonde measurements and forecasts," J. of Geophysical Research, Vol. 111, 2006

[1] E. Georgieva, E. Wilson, M. Miodek, W. Heaps, "Total Column Oxygen Detection using Fabry-Perot Interferometer," Optical Engineering, (2006)

[2] C. L. Korb, C. Y. Weng, "Differential absorption lidar technique for measurement of the atmospheric pressure profile", Applied Optics, Vol. 22, No. 23, 3759-3770, (1983)

[3] K. J. Ritter, "A High Resolution Spectroscopic Study of Absorption Line Profiles in the A-Band of Molecular Oxygen", Dissertation submitted to the University of Maryland Graduate School, (1986)

[4] G. K. Schwemmer, M. Dombrowski, C. L. Korb, J. Milrod, H. Walden, R. H. Kagann, "A lidar system for measuring atmospheric pressure and temperature profiles", Rev. Sci. Instrum. 58 (12), 2226-2237, (1987)

[5] C. L. Korb, G. K. Schwemmer, M. Dombrowski, C. Y. Weng, "Airborne and ground based lidar measurements of the atmospheric pressure profile" Applied Optics, Vol. 28, No. 15 (1989)

[6] J. V. Iribarne, "Atmospheric Thermodynamics" Springer, 1981

[7] W. Demtroder, "Laser Spectroscopy," Springer-Verlag, Berlin, 1982

[8] Rothman LS, Jacquemart D, Barbe A, Benner DC, Birk M, Brown LR, Carleer MR, Chackerian C, Chance K, Coudert LH, Dana V, Devi VM, Flaud JM, Gamache RR, Goldman A, Hartmann JM, Jucks KW, Maki AG, Mandin JY, Massie ST, Orphal J, Perrin A, Rinsland CP, Smith MAH, Tennyson J, Tolchenov RN, Toth RA, Vander Auwera J, Varanasi P, Wagner G., "The HITRAN 2004 molecular spectroscopic database.", Journal of Quantitative Spectroscopy \& Radiative Transfer, 96 (2), p. 139 (2005).

[9] C. N. Flamant, G. K. Schwemmer, C. L. Korb, K. D. Evans, S. P. Palm, "Pressure Measurements using and Airborne Differential Absorption Lidar. Part I: Analysis of the Systematic Error Sources" J. of Atmospheric and Oceanic Technology, Vol. 16, 561-574, (1999)
[10] J. A. Silver and A. C. Stanton, "Optical interference fringe reduction in laser absorption experiments," Appl. Opt. 27, 1914-1916, 1988.

[11] C. R. Webster, "Brewster-plate spoiler: a novel method for reducing the amplitude of interference fringes that limit tunable-laser absorption sensitivities," J. Opt. Soc. Am. B 2, 1464-1470, 1985.

[12] M. A. Stephen, M. A. Krainak, G. R. Allan, H. Riris, "Narrow-band, Tunable, Frequency-doubled, Erbiumdoped Fiber-amplified Transmitter," Opt. Lett., 32, 2073 2075 (2007).

[13] C. E. Dilley, M. A. Stephen, M. P. Savage-leuchs, "High SBS-threshold, narrowband, erbium co-doped with ytterbium fiber amplifier pulses frequency-doubled to 770 nm”, Optics Express, Vol. 15, Issue 22, pp. 14389-14395

\section{BIOGRAPHY}

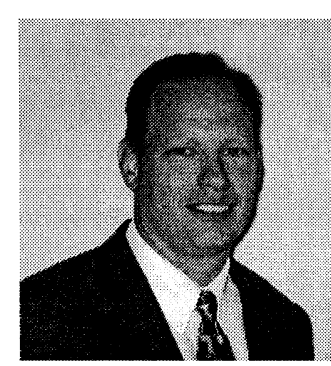

Mark A. Stephen completed his BS in Physics at the University of Delaware in 1992. He completed his MS in Applied Physics at the University of Maryland, Baltimore County in 2002, where he is presently working on his $\mathrm{PhD}$ (also in Applied Physics.) He has been employed at NASA's Goddard Space Flight Center since 1991 where he is a research scientist in the Laser and Electro-Optics Branch. His research activities have included optics and laser components and instruments. $\mathrm{He}$ has also worked on several satellite programs including: the Geoscience Laser Altimeter System, the Mercury Laser Altimeter and the Lunar Orbiter Laser Altimeter. He has done research on diode-pumped Nd:YAG laser systems with an emphasis on laser diode array pumps. He is presently doing research investigating the use of fiber amplifier technology for gas detection using laser spectroscopy. 